\title{
Measurement of the performances of a transparent closed loop two-phase thermosyphon
}

\author{
B. Agostini \& M. Habert \\ ABB Switzerland Ltd., Corporate Research, Baden-Daettwil, Switzerland
}

\begin{abstract}
This paper presents measurements performed on a transparent gravity driven twophase loop. For this purpose a small channel thermosyphon was manufactured completely out of borosilicate glass and equipped with a $100 \mathrm{~nm}$ semi-transparent ITO layer as a direct current heating evaporator. Thus flow patterns could be observed in the evaporator and at the same time the total thermal resistance was calculated with the heater temperature. Influence of heat flux and filling ratio on the thermal resistance and pressure oscillations were investigated.
\end{abstract}

\section{Introduction}

Gravity driven two-phase loops (or thermosyphons) are very widespread devices the nuclear industry, solar heating, computer cooling and chemical engineering, but their use for thermal management of power electronics has been recognised only lately. However, articles on this application are becoming more and more frequent in the literature.

Thermosyphon heat-exchangers allow the use of dielectric fluids and pumpless operation and still benefit from very high heat transfer coefficients. For example Perpina et al [1] recently published an article investigating thermal cycles of thermosyphon cooled traction IGBT modules. Thermosyphons using small channels are particularly appealing for their compactness and low material and fluid use.

Although few data are available with the new dielectric fluids at the required working temperatures and channel diameters, available studies on different thermosyphon configurations are helpful to outline some trends. 
An issue in such systems are the flow instabilities, in particular with small diameter channels. Garrity et al [2] have measured the flow instabilities in square mini-channel thermosyphons (1 mm) filled with HFE7100, for different heights. They concluded that static instabilities are largely influenced by the height of the condenser and stochastic variations in flow rate. Khodabandeh and Furberg [3] have studied the occurrence of instabilities as a function of the channel size. Their experimental results show that flow instabilities increase as channel size decreases. Instabilities occur both at low and very high heat fluxes, with different frequencies and amplitudes for different channel geometries. But the two largest channel diameters trigger less fluctuations.

The performances of a two-phase thermosyphon loop with a small diameter channel evaporator have been recently measured by Khodabandeh [4, 5]. The evaporator channels diameter ranged from 1.1 to $3.5 \mathrm{~mm}$, but the riser had a diameter of $6.1 \mathrm{~mm}$. The heat transfer coefficient was found to be strongly dependant on heat flux and the pressure drop to be adequately predicted by the homogeneous model.

The influence of the filling ratio is also a crucial parameter. Ong and Haider-EAlahi [6] investigated this effect on a R134a filled thermosyphon and found that the performances increased with the filling ratio, reaching a maximum at about $80 \%$ Abou-Ziyan et al [7] found a maximal performance at a filling ratio of 50\%, also with a R134a filled thermosyphon. Furthermore they showed that vibrations could act against the boiling limit and extend the operating range of the thermosyphon. MacDonald et al [8] measured experimentally an optimum at 35\% of filling ratio with refrigerant $\mathrm{R} 11$ in a large diameter tube two-phase thermosyphon and also showed with a numerical model that this optimum was strongly dependent on the respective lengths of the evaporator, riser and condenser. This last fact might explain why Ong and Haider-E-Alahi [6] and Abou-Ziyan et al [7], having different evaporator, riser and condenser lengths, found different optimal filling ratios. Khazaee et al [9] have investigated the influence of filling on the behaviour of geyser boiling in a two-phase closed thermosyphon and discovered that by increasing the filling, the period of geyser boiling and the intensity of temperature oscillation increased, but when the filling becomes less than $30 \%$, the geyser boiling disappears.

To complete this knowledge, a transparent closed loop thermosyphon with a large height over diameter ratio $(>120)$ was manufactured. The evolution of the heater temperature, fluid pressure and instabilities with the heat load and filling ratio were investigated, and flow patterns in the evaporator were observed.

The principle of a small channel thermosyphon is shown in Fig. 1. In such a system working at high heat flux, several assumptions, supported by recent literature, can be made. The dominant flow pattern at low mass velocity in small diameter channels on a large vapor quality range is elongated confined bubble flow. Therefore, these elongated and confined bubbles growing in the vertical evaporator will push liquid plugs upwards towards the condenser. Thus an homogeneous flow can be assumed in a small channel thermosyphon. In this respect, a small channel thermosyphon will essentially behave like a pumped system with a very low mass 


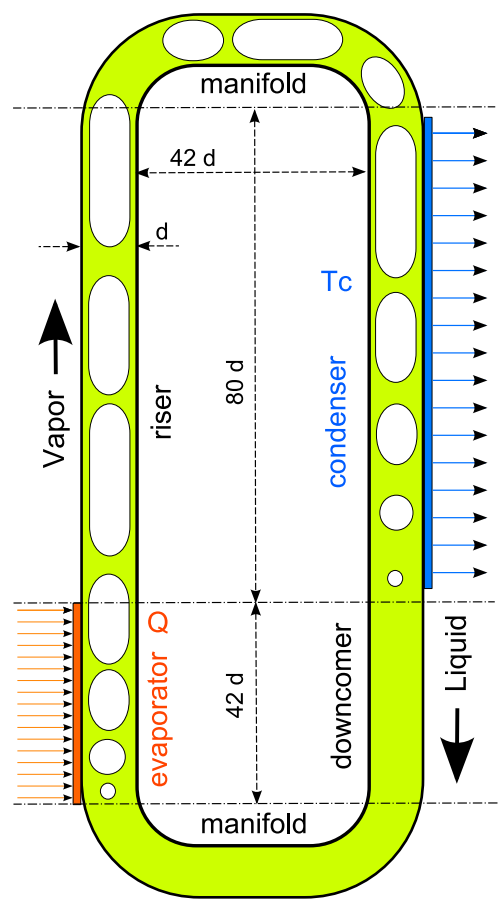

Figure 1: Schematic of the gravity driven two phase loop.

flow rate. This behaviour is quite different from a large channel thermosyphon in which there is little liquid entrainment at the exit of the evaporator and only vapor reaches the condenser. On the contrary, in a small channel thermosyphon the coupling between vapor and liquid is expected to be total and the flow to be homogeneous.

\section{Measurements and methods}

The experimental setup is shown in Fig. 2. A $2.4 \mathrm{~mm}$ internal diameter channel closed loop thermosyphon was manufactured completely out of borosilicate glass and equipped with a $100 \mathrm{~nm}$ semi-transparent ITO (Indium Titanium Oxide) layer as a direct current heating evaporator (160 $\Omega$ electrical resistance). The working fluid was Novec649, chosen because of its low global warming potential (about 1). The loop was equipped with two valves, one at the bottom and one at the top. The bottom valve was used to measure the internal pressure. The pressure oscillations were also measured, during a period of $30 \mathrm{~s}$ in order to catch the minimum and maximum. The top one was used to change the filling of the loop. The electrical current was applied through two electrodes clamped on a sliver padding that was applied on a $2.5 \mathrm{~mm}$ long section of the ITO layer at the bottom and top of the evaporator. The temperature was measured with a type $\mathrm{K}$ thermocouple 
soldered on the top electrode (this is were the highest temperature, and hence the working limit, is expected because of dry-out). The vapor was condensed with a standard Liebig condenser. The coolant was a water-glycol mixture kept at constant temperature with a standard CPU liquid cooling unit. The mass flow of water was kept high enough so that the temperature change through the condenser is negligible. Thus the condensation occurred at constant temperature.

The total thermal resistance was calculated with the following formula:

$$
R_{\mathrm{th}}=\frac{T_{\mathrm{h}}-T_{\mathrm{c}}}{Q}
$$

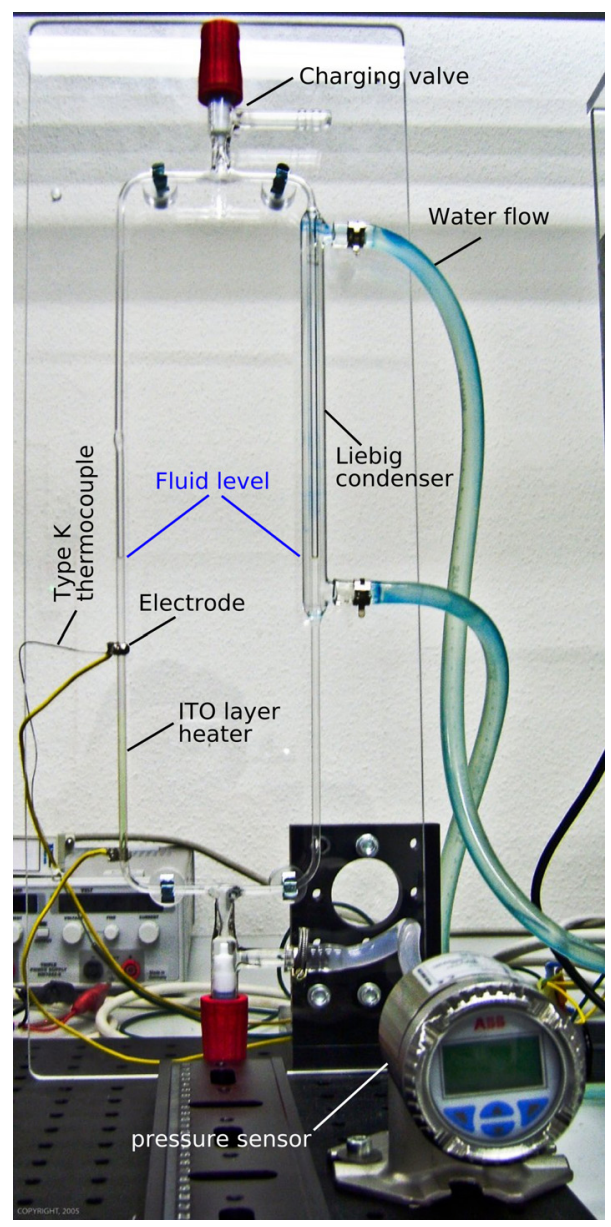

Figure 2: Photography of the experimental setup. 
Table 1: Experimental parameters.

\begin{tabular}{lll}
\hline Variable & Description & Value \\
\hline$D$ & internal diameter & $2.4 \mathrm{~mm}$ \\
$e$ & tube wall thickness & $0.8 \mathrm{~mm}$ \\
$F$ & Filling ratio & $44-78.3 \%$ \\
$L_{\mathrm{c}}$ & condenser length & $188 \mathrm{~mm}$ \\
$L_{\mathrm{e}}$ & evaporator length & $100 \mathrm{~mm}$ \\
$M_{\mathrm{c}}$ & coolant flow rate & $0.75 \mathrm{lmin}$ \\
$Q$ & heating electrical power & $5-35 \mathrm{~W}$ \\
$T_{\mathrm{c}}$ & coolant temperature & $24-25 \mathrm{C}$ \\
$W$ & loop width & $96 \mathrm{~mm}$ \\
\hline
\end{tabular}

and the thermal conductance is simply defined as $U_{\mathrm{th}}=1 / R_{\mathrm{th}}$. The uncertainty on the thermal resistance was calculated with

$$
\frac{\Delta R_{\mathrm{th}}}{R_{\mathrm{th}}}<\frac{\Delta Q}{Q}+\frac{\Delta T_{\mathrm{h}}+\Delta T_{\mathrm{c}}}{T_{\mathrm{h}}-T_{\mathrm{c}}},
$$

yielding a maximal uncertainty comprised between $\pm 7 \%$ and $\pm 27 \%$ at the highest and lowest heat flux respectively.

\section{Analysis of measurements}

Figure 3 shows the heat flux versus the temperature difference between the heater and coolant. The heat flux varies approximately like $\Delta T^{3 / 2}$, while well known pool boiling correlations would suggest $\Delta T^{3}$. However $\Delta T$ starts deviating significantly from this curve at high heat flux and low filling ratios suggesting a strong influence of dry-out, which is not taken into account in pool boiling correlations. The deviation at low heat flux is probably due to the large uncertainty.

Figure 4 shows the thermal conductance versus the heat flux for decreasing filling ratios. It can be seen that for fillings above $54 \%, U_{\text {th }}$ is always increasing with $q$ and independent of the filling. For lower fillings there is a threshold heat flux, function of the filling ratio, above which the effect of $q$ disappears and $U_{\text {th }}$ remains constant. This behaviour is due to the occurrence of intermittent dry-out as shown by Agostini et al [10].

Figure 5 shows the thermal resistance versus filling ratio for increasing heat fluxes. It is merely a different representation of Fig 4. At low heat flux $(<25$ $\mathrm{kW} / \mathrm{m}^{2}$ ), the thermal resistance is slightly increasing with the filling and strongly decreasing with increasing heat flux. At higher heat fluxes, the thermal resistance shows a minimum around $68 \%$ of filling ratio and does not depend on the heat flux at low filling ratios. The presence of a minimum is due to the competition between 
232 Advanced Computational Methods and Experiments in Heat Transfer XI

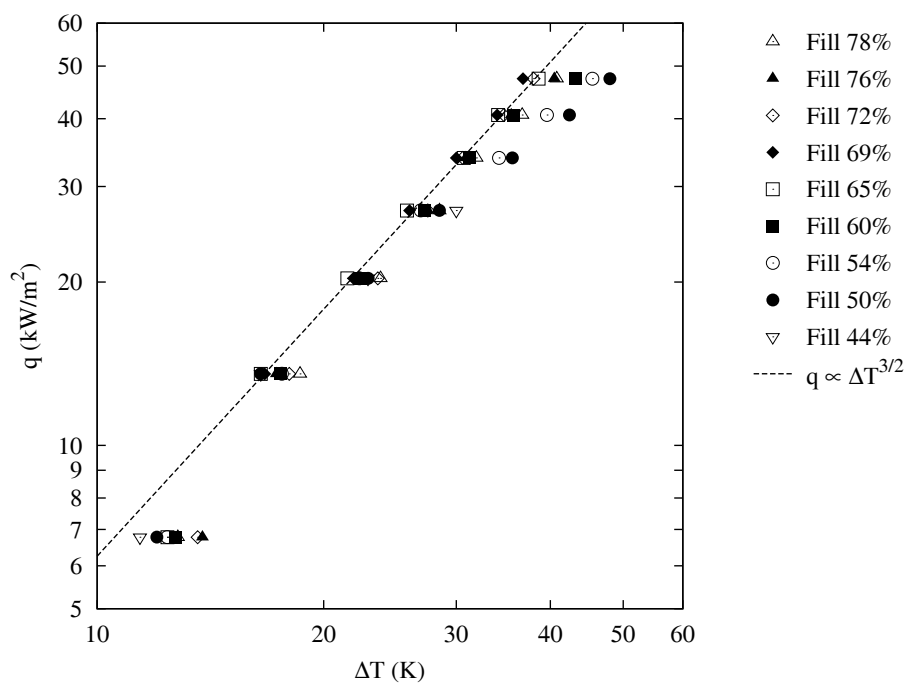

Figure 3: Heat flux versus heater-water temperature difference for decreasing filling ratios.

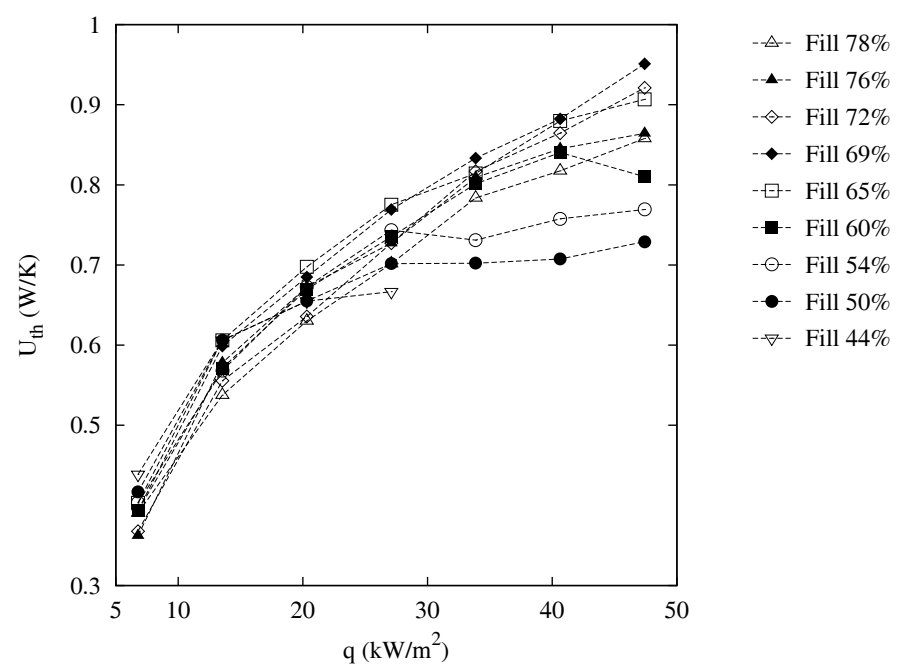

Figure 4: Thermal conductance versus heat flux for decreasing filling ratios. 
Table 2: Measuring devices and accuracy.

\begin{tabular}{lllll}
\hline Measurement & Device & Range & Accuracy & Unit \\
\hline Power & Christ CPM138 wattmeter & $5-35$ & $0.05-0.35$ & $(\mathrm{~W})$ \\
$\begin{array}{l}\text { Heater } \\
\text { temperature }\end{array}$ & TC type K + JUMO dieco & $24-73$ & \pm 1.5 & $(\mathrm{C})$ \\
$\begin{array}{l}\text { Water } \\
\text { temperature }\end{array}$ & Zalman Reserator XT cooler & $24-25$ & \pm 1.5 & $(\mathrm{C})$ \\
Pressure & ABB 261AS & $44-207$ & \pm 1 & $(\mathrm{kPa})$ \\
\hline
\end{tabular}

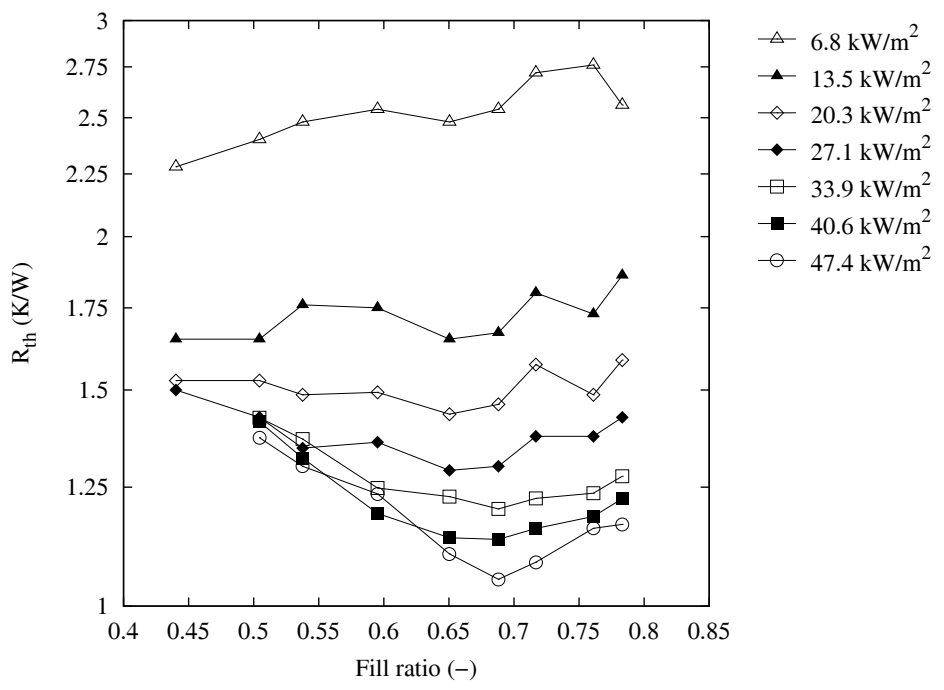

Figure 5: Thermal resistance versus filling ratio for increasing heat fluxes.

the evaporator, which performs better with more liquid and the condenser, which performs better with more vapor.

Figure 6 shows the time averaged saturation pressure versus filling ratio for increasing heat fluxes. As expected the pressure increases with both the filling ratio and the heat flux. However, at high heat flux, the pressure versus filling shows a local minimum in the high filling ratio region.

This distinctive shape is very similar to the characteristic pump curve that explains Ledinegg instabilities shown in Fig. 7. In the present case increasing the filling ratio leads to a flow rate increase for a given heat flux. Thus the presence of this local minimum in the pressure curve might be explained by Ledinegg instabilities.

This is confirmed by the fact that the measured pressure is not constant and undergoes severe and periodic oscillations during the thermosyphon operation, 


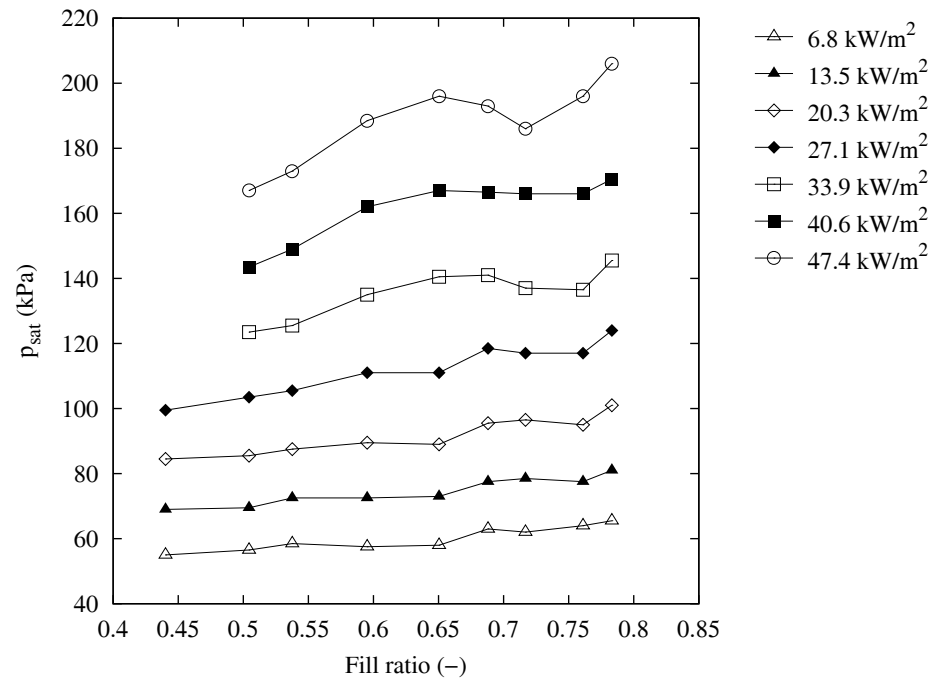

Figure 6: Saturation pressure versus filling ratio for increasing heat fluxes.

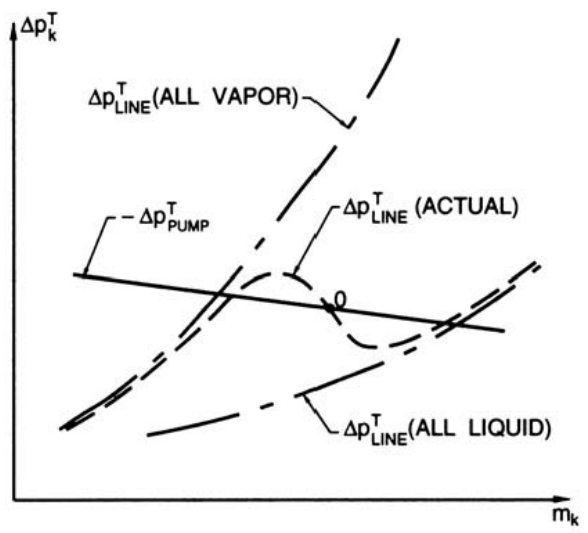

Figure 7: Illustration of Ledinegg instability by Brennen [11].

up to $\pm 13 \%$ of the absolute pressure. These were recorded and are shown on Fig. 8. The pressure oscillations increase with the heat flux, which is consistent with previous literature observations. The influence of the filling ratio is more surprising. At high heat flux in particular, the pressure fluctuations seem to show a peak at around $68 \%$ filling, which corresponds to the filling ratio found at the minimum of the thermal resistance.

Figure 3 shows the different flow patterns that were observed through the semitransparent ITO layer of the evaporator during this experimental campaign. They are arranged from (a) to (i) in increasing heat flux order, for various filling ratios. 


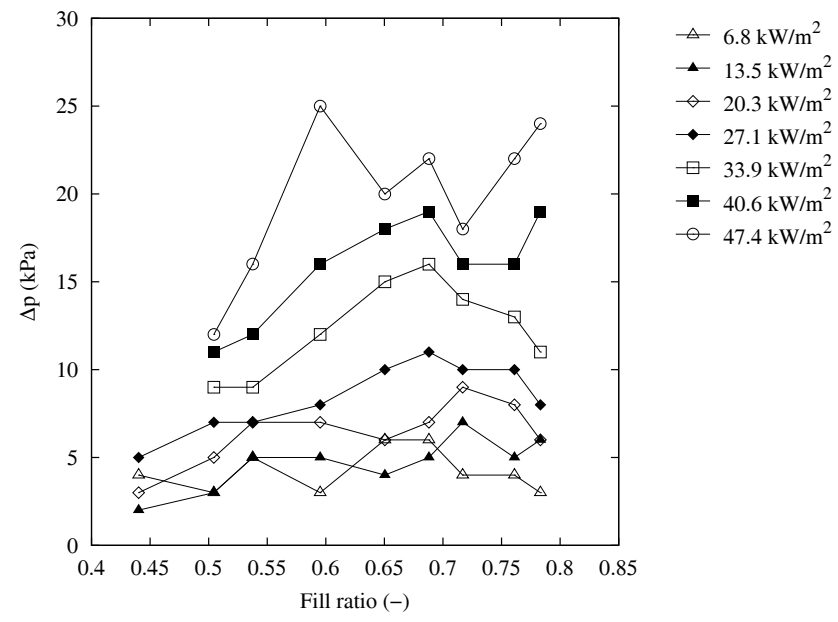

Figure 8: Pressure oscillations versus filling ratio for increasing heat fluxes.

The purpose of this figure is merely to show all possible flow patterns in this type of small channel thermosyphon. The various patterns that were observed are the following:

(a) Free bubbly flow. Small bubbles flow freely without coalescing.

(b) Coalescing bubbly flow. Small bubbles start to coalesce to form larger bubbles at the top.

(c) Bubbly-slug flow. Bubbles coalesce at the bottom and form elongated bubbles at the top.

(d) Slug-churn flow. Elongated bubbles are formed at the bottom and coalesce into a churn flow at the top.

(e) Slug-annular flow. Elongated bubbles are formed at the bottom and coalesce into an annular flow at the top.

(f) churn-annular flow. Elongated bubbles are coalescing at the bottom into a churn flow that becomes annular at the top.

(g) Wavy-annular. An annular flow with surface waves is observed from the bottom to the top.

(h) Smooth-annular flow. An annular flow with a smooth surface is observed from the bottom to the top.

(i) Dry-out. The smooth annular flow is observed at the bottom, but the liquid film disappears intermittently at the top. The following step is a steady disappearance of the film with destruction of the heater.

\section{Conclusions}

A transparent closed loop two-phase thermosyphon was built with a large height over diameter ratio. The total thermal resistance, saturation pressure and pressure 


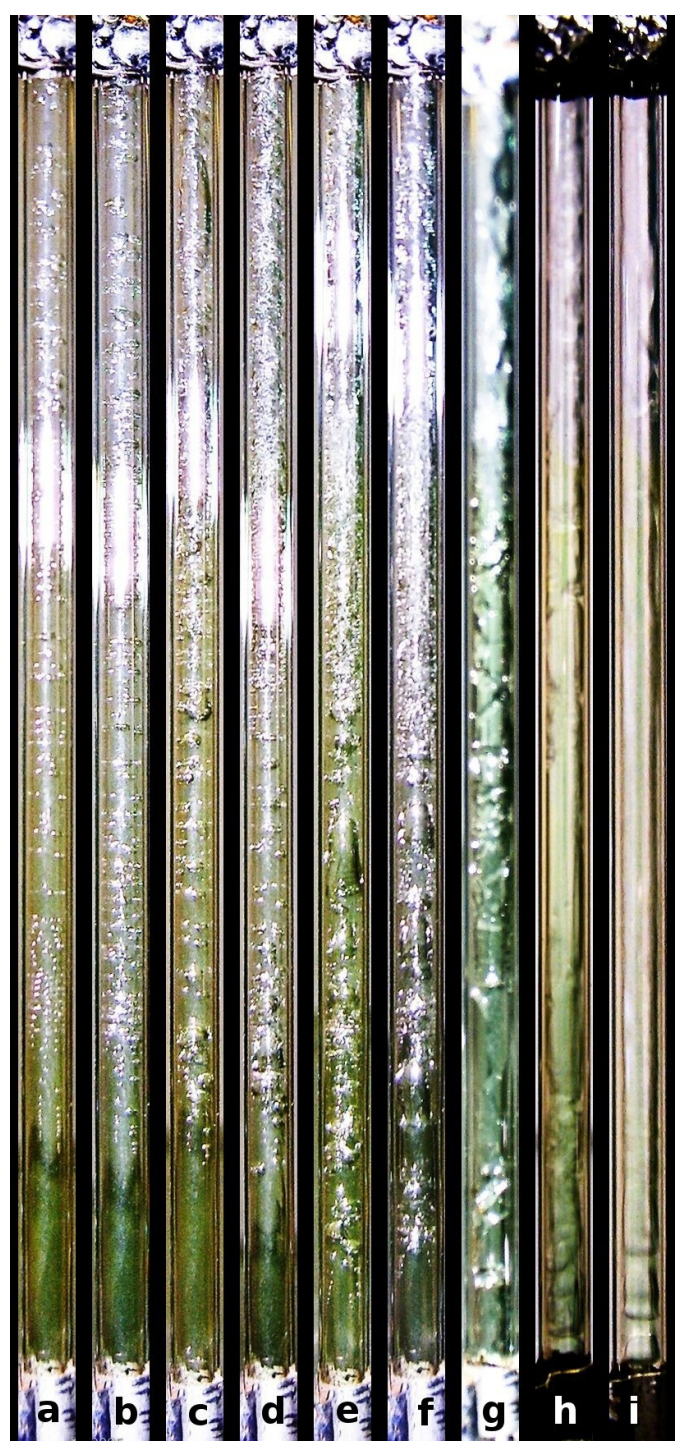

Figure 9: Flow patterns observed through the semi-transparent evaporator for increasing heat fluxes.

oscillations were measured for various heat fluxes and filling ratios. The thermal resistance was found to exhibit a minimum with the filling ratio at about $68 \%$, but only at high heat flux. As expected the thermal resistance is decreasing with increasing heat flux, except at low filling ratio and high heat flux were intermittent dry-out occurs and this dependency disappears. The two-phase loop was found to undergo severe pressure oscillations and a Ledinegg type pressure curve was 
measured. Future improvements of this setup will include laser measurements of bubble velocities in order to estimate the fluid mass flow rate and the oscillation frequencies. Infrared measurement of the heater temperature will also be investigated.

\section{Nomenclature}

$\begin{array}{llllll}D & \text { tube diameter } & (\mathrm{m}) & Q & \text { heat load } & (\mathrm{W}) \\ e & \text { wall thickness } & (\mathrm{m}) & q & \text { heat flux } & \left(\mathrm{kW} / \mathrm{m}^{2}\right) \\ L_{\mathrm{c}} & \text { condenser length } & (\mathrm{m}) & R_{\mathrm{th}} & \text { thermal resistance } & (\mathrm{K} / \mathrm{W}) \\ L_{\mathrm{e}} & \text { evaporator length } & (\mathrm{m}) & T_{\mathrm{c}} & \text { coolant temperature } & (\mathrm{K}) \\ M_{\mathrm{c}} & \text { coolant flow rate } & (\mathrm{l} / \mathrm{min}) & T_{\mathrm{h}} & \text { heater temperature } & (\mathrm{K}) \\ U_{\text {th }} & \text { thermal conductance } & (\mathrm{W} / \mathrm{K}) & W & \text { loop width } & (\mathrm{m})\end{array}$

\section{References}

[1] Perpiñà, X., Piton, M., Mermet-Guyennet, M., Jordà, X. \& Millán, M., Local thermal cycles determination in thermosyphon-cooled traction igbt modules reproducing mission profiles. Microelectronics Reliablity, 47(9-11), pp. 1701-1706, 2007.

[2] Garrity, P., Klausner, J. \& Mei, R., Instability phenomena in a twophase microchannel thermosyphon. International Journal of Heat and Mass Transfer, 52, pp. 1701-1708, 2009.

[3] Khodabandeh, R. \& Furberg, R., Instability, heat transfer and flow regime in a two-phase flow thermosyphon loop at different diameter evaporator channel. Applied Thermal Engineering, In press, pp. xxx-xxx, 2010.

[4] Khodabandeh, R., Heat transfer in the evaporator of an advanced two-phase thermosyphon loop. International Journal of Refrigeration, 28, pp. 190-202, 2005.

[5] Khodabandeh, R., Pressure drop in riser and evaporator of an advanced twophase thermosyphon loop. International Journal of Refrigeration, 28, pp. 725-734, 2005.

[6] Ong, K. \& Haider-E-Alahi, M., Performance of a R-134-a-filled thermosyphon. Applied Thermal Engineering, 23, pp. 2373-2381, 2003.

[7] Abou-Ziyan, H., Helali, A., Fatouh, M. \& El-Nasr,M.A., Performance of stationary and vibrated thermosyphon working with water and R134a. Applied Thermal Engineering, 21, pp. 813-830, 2001.

[8] MacDonald, T., Hwang, K. \& Diciccio, R., Thermosyphon loop performance characteristics: Part 1. experimental study. ASHRAE Trans, 83(2467), pp. 250-259, 1997.

[9] Khazaee, I., Hosseini, R. \& Noie, S., Experimental investigation of effective parameters and correlation of geyser boiling in a two-phase closed thermosyphon. Applied Thermal Engineering, 30, pp. 406-412, 2010.

[10] Agostini, B., Thome, J., Fabbri, M. \& Michel, B., High heat flux two-phase 
238 Advanced Computational Methods and Experiments in Heat Transfer XI

cooling in silicon multimicrochannels. IEEE Transactions on Components and Packaging Technologies, 31(3), pp. 691-701, 2008.

[11] Brennen, E., Fundamentals of Multiphase Flows, Cambridge University Press, pp. 344-364, 2005. 